\title{
Evaluation of shRNA-mediated gene silencing by electroporation in LPB fibrosarcoma cells
}

\author{
Suzana Mesojednik, Urška Kamenšek, Maja Čemažar \\ Department of Experimental Oncology, Institute of Oncology Ljubljana, Slovenia
}

\begin{abstract}
Background. Silencing oncogenes or other genes that contribute to tumor malignancy and progression offers a promising approach to treating cancer. Specific and efficient silencing of gene expression can be achieved by RNA interference (RNAi) technology using small interfering RNA (siRNA) or short hairpin RNA (shRNA). However, a major challenge in RNAi technology is effective delivery of interfering molecules into target cells. The aim of our study was to evaluate electroporation as a perspective method for efficient in vitro transfection of LPB fibrosarcoma cells with plasmid DNA expressing shRNA.

Methods. Induction of shRNA-mediated gene silencing by electroporation was determined by fluorescence microscopy, flow cytometry and western blot analysis. The effect of electroporation conditions on cell survival and proliferation was determined by clonogenic assay.

Results and conclusions. Our results demonstrated that electroporation is a feasible and effective method for delivery of plasmid DNA expressing shRNA into cancer cells in vitro. Electrotransfection of murine LPB fibrosarcoma cells, continuously expressing green fluorescence protein - GFP $\left(L P B_{G F P}\right)$, with plasmid DNA encoding shRNA-GFP, reduced GFP expression, which was determined on the protein level, as well as by measurement of GFP fluorescence intensity. A pronounced reduction in GFP expression level was detected from the second to the fifth day after treatment. Moreover, the method is easy to perform and showed low cell damaging effects, which are the most important and preferential factors for further in vivo studies.
\end{abstract}

Key words: electroporation; plasmid DNA; shRNA; fibrosarcoma.

\section{Introduction}

RNA interference (RNAi) is a newly described natural biological phenomenon mediated by short RNA molecules, which target complementary mRNA. The RNAi pathway is activated by a double-stranded RNA (dsRNA), which is then processed by

Received 8 May 2008

Accepted 22 May 2008

Correspondence to: Dr. Maja Čemažar, Department of Experimental Oncology, Institute of Oncology Ljubljana, SI-1000 Ljubljana, Slovenia. E-mail: mcemazar@onko-i.si the cytoplasmatic enzyme Dicer (RNase III family) into short RNA fragments of 20-21 base pairs. One of the strands becomes incorporated into a RNA-induced silencing complex (RISC), where it serves as a guide for mRNA degradation or downregulation of gene expression. ${ }^{1}$ The process has evolved in eukaryotic organisms as a defense mechanism against viruses and a regulatory mechanism of cellular gene expression. ${ }^{2}$ When the dsRNA is exogenous (viruses), the RNA is imported directly into cytoplasm and cleaved by Dicer to siRNA fragments. Endogenously expressed dsR- 
NAs are processed in the cell nucleus from primary transcripts called pri-miRNA to short, stem-loop structures called pre-miRNA. The dsRNA portion of this pre-miRNA is then bound and cleaved in the cytoplasm by Dicer to produce mature, short, single stranded miRNA that can be integrated into the RISC complex. Downstream of this initial processing, miRNA and siRNA share the same cellular mechanism. ${ }^{1}$ RNAi triggered molecules can be easily synthesized in the lab and used as a research tool for studying gene function and biochemical pathways, as well as for application in gene therapy of human diseases such as viral infections, inflammations and cancer. ${ }^{2-8}$ Different synthetic forms of short RNAs have already been used: siRNA (short interfering RNA) ${ }^{9-11}$, shRNA (short hairpin RNA) ${ }^{12-14}$ and miRNA (microRNA). ${ }^{15}$ The first can be delivered directly into the cytoplasm, where it mimics the natural Dicer product. Synthetic shRNA and miRNA mimic naturally occurring nuclear premiRNA molecules. In order to be delivered into the nucleus, they must be introduced by viral or non-viral vectors. Once in the nucleus, they can be amplified by transcription, thus allowing the gene silencing to be long-term. ${ }^{16}$

Since our understanding of deregulated genes in cancers has grown significantly, RNAi technology has been applied to this field with great promise and enthusiasm all over the world. It has been used to interfere with neoangiogenesis, cell division, inhibition of apoptosis, resistance to chemotherapy and inhibition of anticancer immune responses. ${ }^{4,6,17,18}$ However, the efficiency and duration of siRNA mediated gene silencing in tumors depends to a large extent on the choice of gene delivery system. ${ }^{19}$ In terms of patient safety, non-viral gene delivery systems (siRNA alone, plasmid DNA, liposome, polyplexes, nanoparticles) are superior to viral vectors. However, they have proved to be less efficient, so they have been used in combination with physical methods such as electroporation, gene gun, ultrasound, hyperthermia or magnetofection. ${ }^{20}$

Electroporation has already been proven to be a feasible and effective method for delivering chemotherapeutic drugs and genes into tumor cells. ${ }^{21-23}$ A variety of therapeutic genes have been delivered into tumor cells by this technique, including diphtheria toxin A (DT-A), interleukins (IL-12, IL-2, IL15, IL-18), granulocyte-macrophage colonystimulating factor (GM-CSF), transcription factor p53, tumor necrosis factor-related apoptosis-inducing ligand (TRAIL/Apo2L), interferon-alpha, endostatin and viral protein $\mathrm{R}(\mathrm{Vpr})$, and all have resulted in a therapeutic level of protein expression. ${ }^{22,24}$ Intratumoral electrotransfer of plasmid DNA encoding the human IL-12, IL-2 or tumor antigens has already reached clinical trials. ${ }^{18,22}$ The therapeutic potential of electrically assisted delivery of siRNA molecules into tumors has also been shown. ${ }^{25-28}$ However, siRNA molecules can be rapidly degraded in the tumor tissue, so plasmids expressing shRNA and miRNA may be used for more prolonged and stable expression of RNAi effector molecules. ${ }^{10,14,29-31}$

In the present study, we tested whether electrically-assisted delivery of plasmid DNA expressing shRNA may result in efficient in vitro transfection of LPB fibrosarcoma cells and, consequently, in a reduction of target protein expression level. To test this hypothesis, we electrotransfected the murine LPB fibrosarcoma cell line that continuously expressed a green fluorescence protein (GFP) with plasmid DNA encoding shRNA directed against GFP. The use of reporter protein GFP allowed us to follow up the siRNA activity by three different methods: direct imaging of green fluorescence under an inverted fluorescence microscope, measuring GFP fluorescence intensity by flow cytometer and determin- 

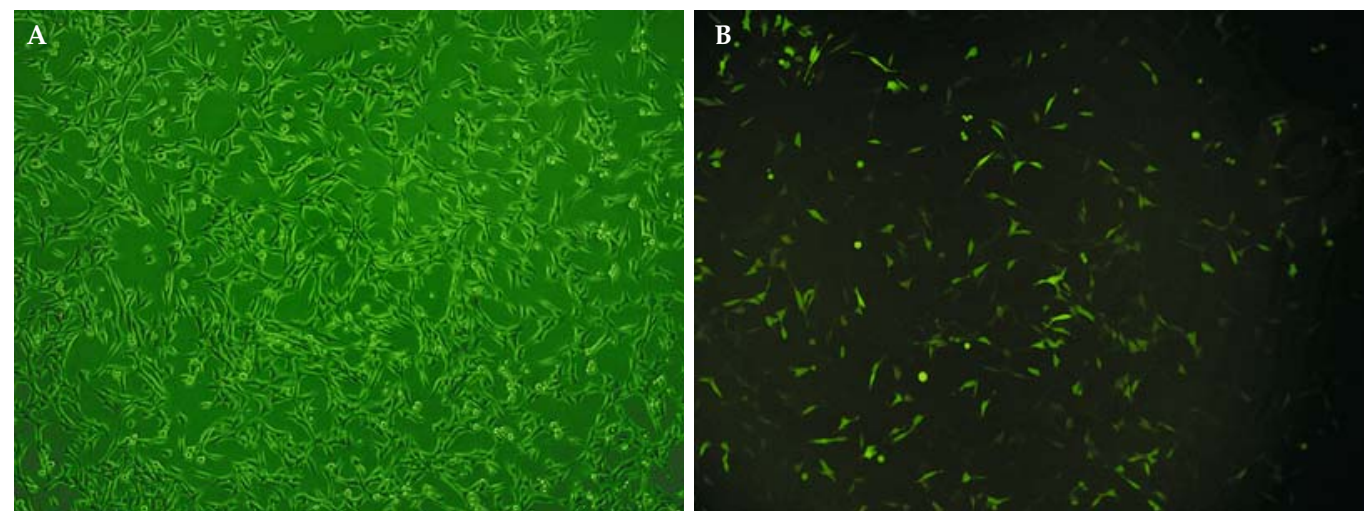

Figure 1. Construction of $\mathrm{LPB}_{\mathrm{GFP}}$ cell line. Light $(\mathrm{A})$ and fluorescent (B) view of $\mathrm{LPB}_{\mathrm{GFP}}$ cells continuously expressing GFP under inverted fluorescence microscope. Magnification: 10×. LPB cells were electrotransfected with psiRNA-EGFP and selected for two months by using $800 \mu \mathrm{g} / \mathrm{ml}$ of geneticin to obtain a cell line that continuously expressed GFP.

ing the changes in protein level by western blot analysis.

\section{Material and methods}

Construction of murine cell line $L P B$ continuously expressing GFP

Murine fibrosarcoma cell line LPB was maintained in Eagle's minimum essential medium (EMEM, Sigma), supplemented with
$10 \%$ fetal calf serum (FCS, Sigma), $2 \mathrm{mM}$ L-glutamine, $1 \mathrm{mM}$ sodium pyruvate, and $100 \mathrm{IU} / \mathrm{ml}$ penicillin/streptomycin (Pliva, Zagreb, Croatia) in a $5 \% \mathrm{CO}_{2}$ humidified incubator at $37^{\circ} \mathrm{C}$.

Plasmid DNA encoding green fluorescence protein (GFP) and neomycin-resistant gene (pEGFP-N1; Clontech, Basingstoke, UK) was used for construction of LPB cell line continuously expressing GFP. Plasmid DNA was amplified in the Top10 DH5 $\alpha$
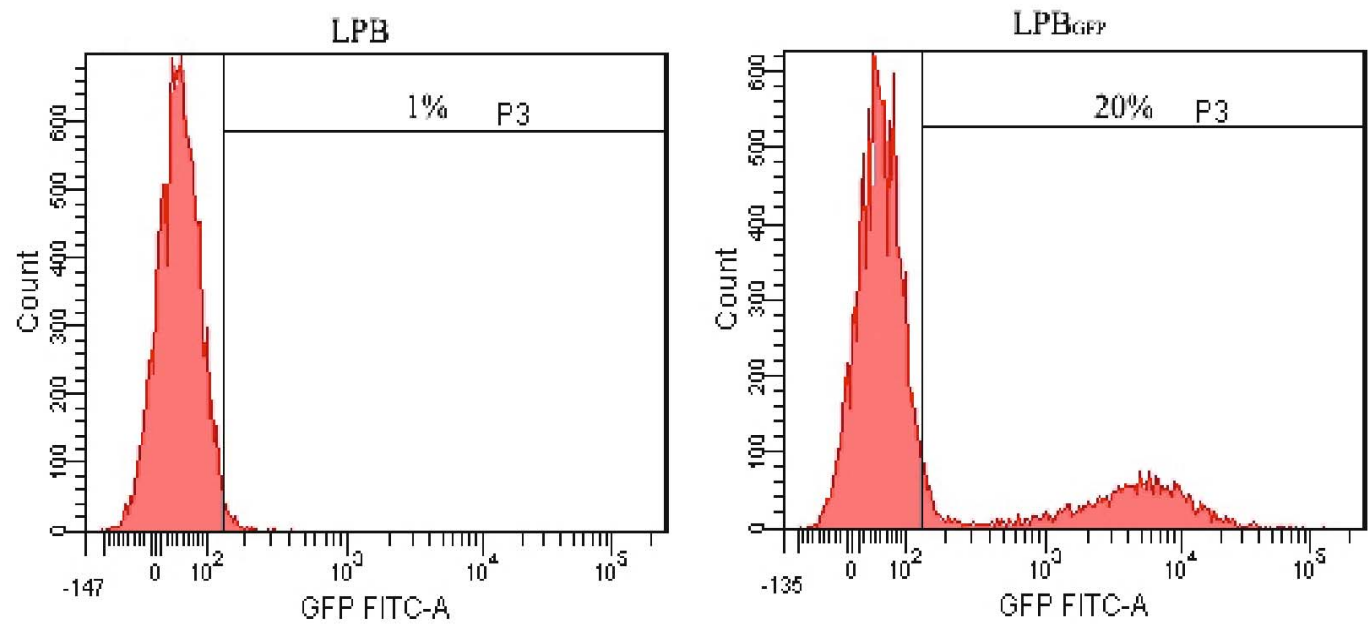

Figure 2. Flow cytometer histograms of GFP fluorescence in $\mathrm{LPB}_{\mathrm{GFP}}$ cells. Flow cytometer analysis showed that only $20 \%$ of cells in $\mathrm{LPB}_{\mathrm{GFP}}$ cell line stably expressed GFP. 
strain of Escherichia coli under kanamycin selection and purified using a QIAGEN Endofree Plasmid Mega Kit (QIAGEN $\mathrm{GmbH}$, Hilden, Germany).

Electroporation was used for introduction of pEGFP-N1 into LPB cells in vitro. Specifically, LPB cells grown as monolayers were harvested and a $2.5 \times 10^{7}$ cells $/ \mathrm{ml}$ cell suspension was prepared in electroporation buffer (125 mM sucrose, $10 \mathrm{mM} \mathrm{K}_{2} \mathrm{HPO}_{4}$, 2,5 mM KH$\left.{ }_{2} \mathrm{PO}_{4}, 2 \mathrm{mM} \mathrm{MgCl} 2 \times 6 \mathrm{H}_{2} \mathrm{O}\right)$. A dense cell suspension with a concentration of $1 \times 10^{6}$ cells and $10 \mu \mathrm{g}$ of pEGFP$\mathrm{N} 1$ in $50 \mu \mathrm{l}$ of electroporation buffer was placed between two flat parallel stainless steel electrodes with a 2-mm gap connected to the GT-1 electroporator (Faculty of Electrical Engineering, Ljubljana, Slovenia) and subjected to eight square-wave electric pulses with an amplitude per distance 600 $\mathrm{V} / \mathrm{cm}, 5 \mathrm{msec}$ duration time and $1 \mathrm{~Hz}$ repetition frequency. After exposure to electric pulses, the cells were incubated for $5 \mathrm{~min}$ at room temperature. Electrotransfection of LPB cells with pEGFP-N1 and a further 2 months culturing of the treated cells under selectable marker geneticin $(800 \mu \mathrm{g} / \mathrm{ml})$ resulted in continuous expression of GFP protein in $20 \%$ of the cells (Figure 1,2). The GFP expressing cell line was named $\mathrm{LPB}_{\mathrm{GFP}}$. This cell line was further used in subsequent experiments of siRNA-mediated gene silencing after electrotransfection of cells with plasmid psiRNA-EGFP.

\section{Electrotransfection of $L P B_{G F P}$ cells with shRNA-expressing $p D N A$}

The above described electroporation protocol was used for the introduction of shRNA-expressing pDNAs, psiRNA-EGFP (Invivogen, San Diego, USA) and psiRNAscramble (Invivogen, San Diego, USA), into $\mathrm{LPB}_{\mathrm{GFP}}$ cells in vitro.

psiRNA-EGFP transcribes a singlestranded RNA 5' - GCA AGC UGA CCC
UGA AGU UCA CCA CCU GAA CUU CAG GGU CAG CUU GCuu - 3', which forms stem-loop-structured siRNA, targeted to EGFP mRNA (targeted sequence: 5' GCA AGC TGA CCC TGA AGT TCA 3'), with a loop sequence of CACC.

psiRNA-scramble which transcribes the non related sequence RNA 5'- GCA UAU GUG CGU ACC UAG CAU UCA AGA GAU GCU AGG UAC GCA CAU AUG Cuu - 3' was used as control pDNA to psiRNA-EGFP throughout the study.

Both shRNA-expressing plasmid DNAs are driven by the CMV enhancer/promoter. Plasmid DNAs expressing shRNA were amplified in the GT116 strain of Escherichia coli under zeocin selection, and purified using a QIAGEN Endofree Plasmid Mega Kit.

\section{Determination of siRNA silencing effect}

Fluorescence microscopy. Electrotransfected cells were plated on 6-cm Petri dishes (Corning Costar, Acton, MA, USA). In $\mathrm{LPB}_{\mathrm{GFP}}$ fibrosarcoma cells, GFP fluorescence was detected by inverted fluorescence microscope. From the first to the fifth day after treatment visible and fluorescence images at 10× magnifications were taken with a CCD Camera (Olympus, Germany) connected to fluorescence microscope.

Flow cytometry. Electrotransfected cells were plated on 10-cm Petri dishes (Corning Costar). From the first to the fifth day after treatment, cells were subjected to flow cytometry analysis. Twenty thousand cells were analyzed for each sample. The threshold for autofluorescence was set to a value at which $99 \%$ of the control cells (LPB cells without GFP) were included. The mean GFP fluorescence intensity of the cells expressing GFP was determined from histograms obtained by flow cytometer (Becton Dickinson, Calibur, Franklin Lakes, USA). The experiments were repeated three times. 

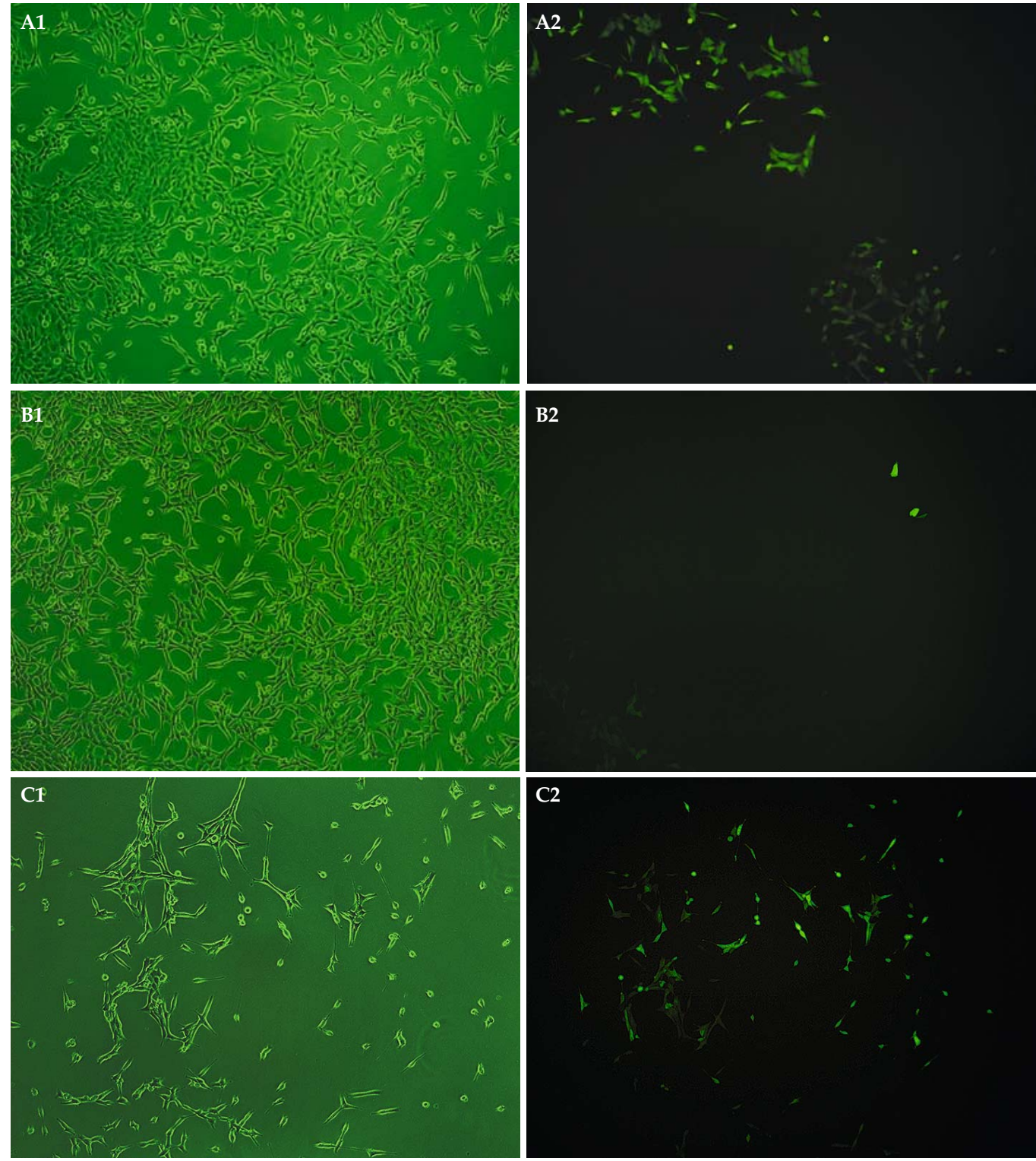

Figure 3. Imaging of GFP expressions in $\mathrm{LPB}_{\mathrm{GFP}}$ cells on the fifth day after treatment under inverted fluorescence microscope. Light (1) and fluorescent view (2) of experimental groups: $\mathrm{A}\left(\mathrm{LPB}_{\mathrm{GFP}}+\mathrm{EP}\right), \mathrm{B}\left(\mathrm{LPB}_{\mathrm{GFP}}+\right.$ psiRNA$\mathrm{EGFP}+\mathrm{EP})$, and $\mathrm{C}\left(\mathrm{LPB}_{\mathrm{GFP}}+\right.$ psiRNA-scr + $\left.\mathrm{EP}\right)$. A pronounced reduction in the fraction of GFP positive cells was observed after electrotransfection of $\mathrm{LPB}_{\mathrm{GFP}}$ cells with psiRNA-EGFP (B2). Magnification 10×. 
Western blot. After electrotransfection, $\mathrm{LPB}_{\mathrm{GFP}}$ cells were plated on $10-\mathrm{cm}$ Petri dishes. Cells were harvested by cell scraper $48 \mathrm{~h}$ after transfection, lysed in lysis buffer (20 mM Tris, mM NaCl, 0.1\% Triton), sonificated for $15 \mathrm{~s}$ and centrifuged for $10 \mathrm{~min}$ $\left(10000 \times \mathrm{g}, 4^{\circ} \mathrm{C}\right)$. The obtained total protein suspension was stored at $-20^{\circ} \mathrm{C}$ for further western blot analysis. The protein concentration was determined by BCA Protein Assay Kit (Pierce, Rockford, USA). The amount of total protein extract from samples was adjusted to $50 \mu \mathrm{g} /$ lane, followed by the transfer of protein samples to a nitrocellulose membrane sheet (Pierce, Rockford, USA). Antibodies against GFP (B-2) (sc-9996, 1: 1000, Santa Cruz Biotechnology INC, Santa Cruz, CA, USA) and HRP-conjugated goat anti-mouse antibodies IgG $_{2 a}-\mathrm{HRP}$ (sc-2061, Santa Cruz Biotechnology INC, Santa Cruz, CA, USA) were used. Protein bands were detected after incubation of the membrane with chemo-luminescent reagent (Santa Cruz Biotechnology INC, Santa Cruz, CA, USA) and 1 minute exposure to film. Optical densities of proteins were determined by the Image J software system (National Institute of Health, Research Services Branch, Bethesda, MD USA).

\section{Effect of treatment on cell survival and proliferation}

The sensitivity of the cell line to plasmid DNAs, electroporation conditions and the combination of them was determined by clonogenic assay. Cells (250 - 500) were plated on 6-cm Petri dishes with $4 \mathrm{ml}$ of medium and incubated in a $5 \% \mathrm{CO}_{2}$ humidified incubator at $37^{\circ} \mathrm{C}$. After 10 to 12 days, colonies were stained with crystal violet and counted. Colonies containing less than 50 cells were ignored. Plating efficiency and the surviving fraction were then calculated. The experiments were performed in triplicate and repeated three times.

\section{Statistical analysis}

The data were tested for normality of distribution using the Kolmogorov-Smirnov test. Differences between experimental groups were statistically evaluated by oneway analysis of variance (ANOVA) followed by the Holm-Sidak test for multiple comparison. A p-value of less than 0.05 was considered to be statistically significant. Statistical analysis was done using Sigma Stat (Systat Software Inc., London, UK)

\section{Results}

Induction of RNAi after electrotransfection of psiRNA-EGFP into $L P B_{G F P}$ cells

Fluorescence microscope imaging showed a markedly reduced level of GFP expression from the second to the fifth day in $\mathrm{LPB}_{\mathrm{GFP}}$ cells after electrotransfection with psiRNAEGFP. No reduction in GFP expression level was observed in $\mathrm{LPB}_{\mathrm{GFP}}$ cells treated with electric pulses only or electrotransfected with control psiRNA-scr (Figure 3).

Flow cytometry measurements were also performed from the first to the fifth day after electrotransfection. The intensities of GFP fluorescence were diminished in $\mathrm{LPB}_{\mathrm{GFP}}$ cells electrotransfected with psiRNA-EGFP in comparison with the control group electrotransfected with psiRNA-scr. The second day after electrotransfection with psiRNA-EGFP, the mean fluorescence intensity of GFP was lower by $14 \pm 6.3 \%$ and from the third to the fifth day after electrotransfection it was around 30\% lower than in the control group. However, the obtained mean fluorescence intensities did not show a statistically significant difference in GFP fluorescence intensities between the experimental and control group (Figure 4).

Western blot analysis of protein optical density showed a $20 \%$ reduction in GFP 


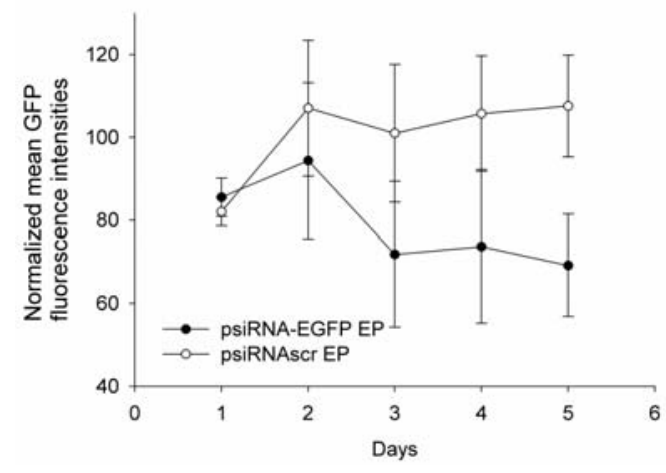

Figure 4. Flow cytometry measurements of GFP fluorescence intensity levels in $\mathrm{LPB}_{\mathrm{GFP}}$ cells after electrotransfection with psiRNA-EGFP or psiRNA-scr over time. Shown are GFP fluorescence intensities normalized for each day to GFP fluorescence intensities of the control group, which was treated with electric pulses only.

protein level on the second day after electrotransfection with psiRNA-EGFP compared to the control (psiRNA-scr) (Figure 5).

\section{Cell survival and proliferation after electrotransfection of psiRNA-EGFP into $L P B_{G F P}$ cells}

$\mathrm{LPB}_{\mathrm{GFP}}$ cells showed a $33 \pm 15 \%$ reduction in colony formation after treatment with electric pulses only. The introduction of psiRNA-EGFP or psiRNA-scr into $\mathrm{LPB}_{\mathrm{GFP}}$ cells by electroporation reduced colony formation even more, by $65 \pm 9.7 \%$ and $70 \pm 11.5 \%$ (Figure 6).

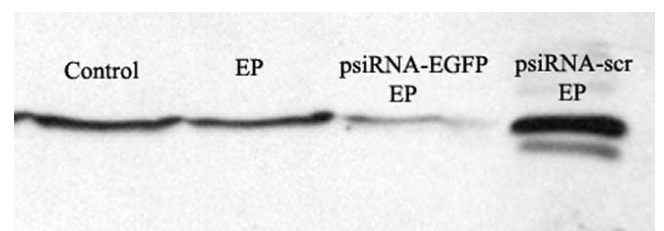

Figure 5. Western blot analyses of the changes in GFP protein level in $\mathrm{LPB}_{\mathrm{GFP}}$ cells after electroporation and electrotransfection with psiRNA-EGFP or pshRNAscr. On day 2 after electrotransfection of $\mathrm{LPB}_{\mathrm{GFP}}$ cells with psiRNA-EGFP, western blot analysis showed up to $20 \%$ lower GFP protein level in comparison with the control vector.

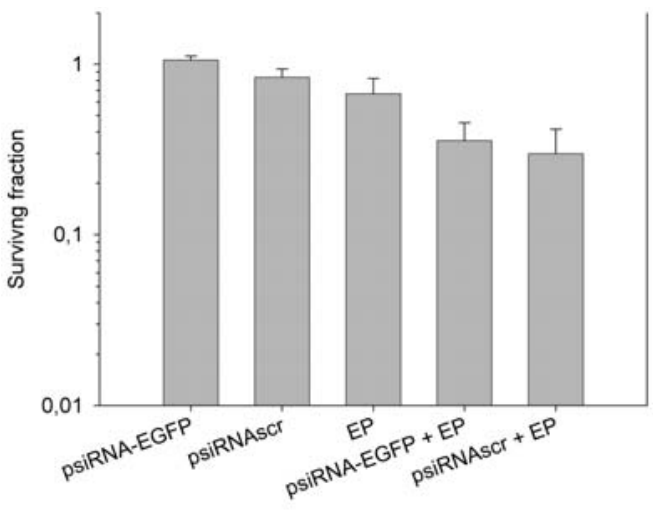

Figure 6. Cell survival after electrotransfection with psiRNA-EGFP or pshRNAscr. Values were normalized to the survival of untreated cells. Data present MEAN \pm $\mathrm{SE}$, which were pooled from triplicates of three experiments.

\section{Discussion}

The study examined whether electroporation as a physical gene delivery method and plasmid DNA as a gene delivery vector could be used for the introduction of shRNA into murine LPB fibrosarcoma cells. The study was performed in vitro in a murine LPB fibrosarcoma cell line constructed in such a way as to continuously express GFP $\left(\mathrm{LPB}_{\mathrm{GFP}}\right)$. Reporter gene GFP was chosen due to the ease of imaging and measuring GFP gene expression levels in $\mathrm{LPB}_{\mathrm{GFP}}$ cells, which enabled the determination of siRNA activity directed against GFP.

Various delivery methods for in vitro gene silencing have been used to date, mostly transduction with adenoviral, retroviral and SV40 vectors, as well as chemical (lipoplexes, polyplexes) non-vector or vector based transfection methods, which have shown a good silencing effect of either reporter or therapeutic genes. ${ }^{9,32-35}$ Among nonviral delivery methods, liposomes have proved to be a very suitable method for in vitro delivery of siRNA or shRNA. ${ }^{15,26,27,29-31}$ In addition, a recent study has demonstrated that electroporation also results in effective 
siRNA mediated gene silencing. Namely, in a study by Merkova et al. electroporation was used to introduce siRNA into chronic myeloid leukemia (CML) cells. Gene expression was reduced by $37 \%$, which is comparable with our results using shRNA (35\% at day 5). However, in both studies, electroporation was associated with higher cell death compared to other delivery methods. ${ }^{15,26,27,29-31,36}$

In our study, the siRNA silencing effect was measured quantitatively and temporally. Compared with the electrotransfected control vector psiRNA-scr, the psiRNAEGFP reduced GFP expression after electrotransfection into $\mathrm{LPB}_{\mathrm{GFP}}$ cells, which was shown by flow cytometry analysis of GFP fluorescence intensity, by western blot analysis of GFP protein levels in cells and by fluorescence microscope imaging of cells expressing GFP. The second day after electrotransfection of $\mathrm{LPB}_{\mathrm{GFP}}$ cells with psiRNA-EGFP, a 14\% decrease in GFP fluorescence intensity was related to a $20 \%$ reduction in GFP protein level. On the third day, siRNA activity decreased GFP fluorescence intensity by $\sim 30 \%$ and this level remained stable for the next two measuring days. Since the duration of gene silencing by naked siRNA is usually less than 5 days in rapidly growing cell lines 28,37 , we assumed that plasmid DNA expressing shRNA had been transferred into the nucleus, where it was amplified by transcription and a prolonged silencing effect was therefore achieved in the rapidly dividing $\mathrm{LPB}_{\mathrm{GFP}}$ cell line (doubling time 24-30 h). No decrease in GFP intensities was determined on the first day after electrotransfection with psiRNA-EGFP, which could be explained by the more than $24 \mathrm{~h}$ half-life of the GFP protein. ${ }^{38}$ An increased reduction in the fraction of cells expressing GFP was observed by fluorescence microscopy from the second to the fifth day after electrotransfection with psiRNA-EGFP, which coincided with the mean GFP fluorescence intensity results. Nevertheless, the mean values of GFP fluorescence between the experimental (psiRNA-EGFP) and control groups (psiRNA-scr) did not show statistically significant differences. This may be because only $20 \%$ of cells in the $\mathrm{LPB}_{\mathrm{GFP}}$ cell line stably expressed GFP. Namely, the cells which did not express GFP and were possibly electrotransfected with psiRNAEGFP contributed to the apparently lower GFP silencing effect. We believe that more convincing GFP silencing after electrotransfection with psiRNA-EGFP may therefore be achieved by using the $\mathrm{LPB}_{\mathrm{GFP}}$ cell line, in which all cells would express GFP. Another factor that could affect the GFP silencing effect is the CMV enhancer/ promoter, which drives shRNA expressing plasmid DNA. It has been shown that CMV enhancer/promoter can be inactivated in cells by methylation, which inhibits transgene expression. Methylation of the promoter may thus be a critical event in gene transcription. ${ }^{39,40}$

The sensitivity of $\mathrm{LPB}_{\mathrm{GFP}}$ cells to electric pulse conditions did not exceed the critical level. Cell proliferation was reduced by only $33 \%$. More concerning is the cytotoxicity of psiRNA-EGFP and even more of psiRNAscr introduced into the cells by electroporation, which resulted in a $65 \%$ and $70 \%$ reduction of colony formation, respectively, in comparison with the untreated control group $\left(\mathrm{LPB}_{\mathrm{GFP}}\right)$. It is probable that LPB cells that had been previously transfected with pEGFP-N1 might have increased sensitivity to electrotransfection by default, since expression of exogenous GFP can affect cell viability. ${ }^{41}$ However, cytotoxicity results are not so relevant in demonstrating the potentiality of electroporation as a technique for efficient delivery of plasmid DNA expressing shRNA into cancer cells, although they should be taken into account when the therapeutic shRNA molecules are tested. 
However, the results of the present study showed that electroporation is feasible and effective method for the in vitro delivery of plasmid DNA expressing shRNA. Further studies should be extended to in vivo experiments examining whether electrically assisted delivery of plasmid DNA expressing shRNA-GFP into $\mathrm{LPB}_{\mathrm{GFP}}$ subcutaneous tumors may reduce GFP expression levels in tumor cells for a sufficient period of time. In vivo electrotransfection of plasmid DNA into tumors has been reported as an efficient gene delivery method, with great potential for cancer treatment. ${ }^{22,23}$ On the other hand, low transfection efficiencies have been obtained in some tumor types with this delivery method, which may be a problem in its application for siRNA mediated cancer gene therapy. ${ }^{42-46}$ However, only a few in vivo studies have demonstrated successful silencing of a target gene after electrotransfection of tumors with plasmid DNA expressing therapeutic shRNA. ${ }^{30,31}$ Reporter genes should be used for validation of the method. Takahashi and coworkers used plasmid DNA expressing shRNA directed against luciferase, whereby luciferase activity was measured over time indirectly. ${ }^{29}$ Namely, tumors were excised and homogenized, the homogenate was centrifuged, the supernatant was then mixed with luciferase assay buffer and the chemiluminiscence produced was measured in a luminometer. The ability to image a reporter gene directly can save significant time and resources, especially in view of the high cost of siRNA molecules and the number of animals required for in vivo studies. Direct imaging of the GFP reporter gene expression profile after treatment would enable non-invasive monitoring of siRNA activity in mice over time. On the basis of GFP reporter gene imaging, we can predict whether electrically assisted delivery of plasmid DNA expressing GFP specific shRNA into tumors in mice is safe, specific and effective, how much plasmid DNA transfer occurred, and whether and for how long the shRNA is expressed in a specific tumor type.

\section{Conclusion}

Our in vitro results showed that electroporation is a feasible and effective method for delivering plasmid DNA expressing shRNA into tumor cells in vitro, which was shown through efficient silencing of the targeted reporter gene for GFP. Imaging and measurements of GFP expression levels over time in vitro showed that electroporation, in combination with plasmid DNA as the gene delivery vector, can induce a prolonged gene silencing effect in rapidly dividing $\mathrm{LPB}_{\mathrm{GFP}}$ cells. Moreover, electroporation proved to be reproducible, easy to perform and showed a low cell damaging effect, which are all important and preferential factors for in vivo experiments and, ultimately for clinical trials.

\section{Acknowledgement}

The authors acknowledge financial support from the state budget by the Slovenian Research Agency (Projects No. P3-0003, J37044, J3-9580). We thank the Department of Cytopathology at the Institute of Oncology, Ljubljana, for help with flow cytometer analysis. We thank Prof. dr. Gregor Serša and Gregor Tevž for critically reading the manuscript and Mira Lavrič for help with experiments. 


\section{References}

1 Agami R. RNAi and related mechanisms and their potential use for therapy. Curr Opin Chem Biol 2002; 6(6): 829-34.

2 Caplen NJ. Gene therapy progress and prospects. Downregulating gene expression: the impact of RNA interference. Gene Ther 2004; 11(16): 1241-8.

3 Ryther RC, Flynt AS, Phillips JA, III, Patton JG. siRNA therapeutics: big potential from small RNAs. Gene Ther 2005; 12(1): 5-11.

4 Izquierdo M. Short interfering RNAs as a tool for cancer gene therapy. Cancer Gene Ther 2005; 12(3): 217-27.

5 Scherer L, Rossi JJ. Recent applications of RNAi in mammalian systems. Curr Pharm Biotechnol 2004; 5(4): 355-60.

6 Pai SI, Lin YY, Macaes B, Meneshian A, Hung CF, $\mathrm{Wu}$ TC. Prospects of RNA interference therapy for cancer. Gene Ther 2006; 13(6): 464-77.

7 Dykxhoorn DM, Palliser D, Lieberman J. The silent treatment: siRNAs as small molecule drugs. Gene Ther 2006; 13(6): 541-52.

8 Jana S, Chakraborty C, Nandi S, Deb JK. RNA interference: potential therapeutic targets. Appl Microbiol Biotechnol 2004; 65(6): 649-57.

9 Bantounas I, Phylactou LA, Uney JB. RNA interference and the use of small interfering RNA to study gene function in mammalian systems. $J \mathrm{Mol}$ Endocrinol 2004; 33(3): 545-57.

10 Behlke MA. Progress towards in vivo use of siRNAs. Mol Ther 2006; 13(4): 644-70.

11 Birmingham A, Anderson E, Sullivan K, Reynolds A, Boese Q, Leake D et al. A protocol for designing siRNAs with high functionality and specificity. Nat Protoc 2007; 2(9): 2068-78.

12 Paddison PJ, Caudy AA, Bernstein E, Hannon GJ, Conklin DS. Short hairpin RNAs (shRNAs) induce sequence-specific silencing in mammalian cells. Genes Dev 2002; 16(8): 948-58.

13 Brummelkamp TR, Bernards R, Agami R. A system for stable expression of short interfering RNAs in mammalian cells. Science 2002; 296(5567): 550-3.

14 Cullen BR. Induction of stable RNA interference in mammalian cells. Gene Ther 2006; 13(6): 503-8.
15 McManus MT, Petersen CP, Haines BB, Chen J, Sharp PA. Gene silencing using micro-RNA designed hairpins. RNA 2002; 8(6): 842-50.

16 McAnuff MA, Rettig GR, Rice KG. Potency of siRNA versus shRNA mediated knockdown in vivo. J Pharm Sci 2007; 96(11): 2922-30.

17 Gottesman MM. Cancer gene therapy: an awkward adolescence. Cancer Gene Ther 2003; 10(7): 501-8.

18 Meyer M, Wagner E. Recent developments in the application of plasmid DNA-based vectors and small interfering RNA therapeutics for cancer. Hum Gene Ther 2006; 17(11): 1062-76.

19 Xie FY, Woodle MC, Lu PY. Harnessing in vivo siRNA delivery for drug discovery and therapeutic development. Drug Discov Today 2006; 11(1-2): 6773.

20 Russ V, Wagner E. Cell and tissue targeting of nucleic acids for cancer gene therapy. Pharm Res 2007; 24(6): 1047-57.

21 Sersa G, Cemazar M, Miklavcic D, Rudolf Z. Electrochemotherapy of tumours. Radiol Oncol 2006; 40(3): 163-74.

22 Heller LC, Heller R. In vivo electroporation for gene therapy. Hum Gene Ther 2006; 17(9): 890-7.

23 Tamura T, Sakata T. Application of in vivo electroporation to cancer gene therapy. Curr Gene Ther 2003; 3(1): 59-64.

24 Cemazar M, Sersa G. Electrotransfer of therapeutic molecules into tissues. Curr Opin Mol Ther 2007; 9(6): 554-62.

25 Fan $Y$, Xin XY, Chen BL, Ma X. Knockdown of RAB25 expression by RNAi inhibits growth of human epithelial ovarian cancer cells in vitro and in vivo. Pathology 2006; 38(6): 561-7.

26 Nakai N, Kishida T, Shin-Ya M, Imanishi J, Ueda Y, Kishimoto $\mathrm{S}$ et al. Therapeutic RNA interference of malignant melanoma by electrotransfer of small interfering RNA targeting Mitf. Gene Ther 2007; 14(4): 357-65.

27 Takei Y, Nemoto T, Mu P, Fujishima T, Ishimoto T, Hayakawa $\mathrm{Y}$ et al. In vivo silencing of a molecular target by short interfering RNA electroporation: tumor vascularization correlates to delivery efficiency. Mol Cancer Ther 2008; 7(1): 211-21. 
28 Golzio M, Mazzolini L, Ledoux A, Paganin A, Izard $\mathrm{M}$, Hellaudais $\mathrm{L}$ et al. In vivo gene silencing in solid tumors by targeted electrically mediated siRNA delivery. Gene Ther 2007; 14(9): 752-9.

29 Takahashi Y, Nishikawa M, Kobayashi N, Takakura Y. Gene silencing in primary and metastatic tumors by small interfering RNA delivery in mice: quantitative analysis using melanoma cells expressing firefly and sea pansy luciferases. J Control Release 2005; 105(3): 332-43.

30 Takahashi Y, Nishikawa M, Takakura Y. Suppression of tumor growth by intratumoral injection of short hairpin RNA-expressing plasmid DNA targeting beta-catenin or hypoxia-inducible factor 1alpha. J Control Release 2006; 116(1): 90-5.

31 Rejiba S, Wack S, Aprahamian M, Hajri A. K-ras oncogene silencing strategy reduces tumor growth and enhances gemcitabine chemotherapy efficacy for pancreatic cancer treatment. Cancer Sci 2007; 98(7): 1128-36.

32 Chiu YL, Rana TM. RNAi in human cells: basic structural and functional features of small interfering RNA. Mol Cell 2002; 10(3): 549-61.

33 Devi GR. siRNA-based approaches in cancer therapy. Cancer Gene Ther 2006; 13(9): 819-29.

34 Thomas M, Lu JJ, Ge Q, Zhang C, Chen J, Klibanov AM. Full deacylation of polyethylenimine dramatically boosts its gene delivery efficiency and specificity to mouse lung. Proc Natl Acad Sci U S A 2005; 102(16): 5679-84.

35 Grayson AC, Doody AM, Putnam D. Biophysical and structural characterization of polyethylenimine-mediated siRNA delivery in vitro. Pharm Res 2006; 23(8): 1868-76.

36 Merkerova M, Klamova H, Brdicka R, Bruchova $\mathrm{H}$. Targeting of gene expression by siRNA in CML primary cells. Mol Biol Rep 2007; 34(1): 27-33.

37 Bartlett DW, Davis ME. Insights into the kinetics of siRNA-mediated gene silencing from live-cell and live-animal bioluminescent imaging. Nucleic Acids Res 2006; 34(1): 322-33.

38 Corish P, Tyler-Smith C. Attenuation of green fluorescent protein half-life in mammalian cells. Protein Eng 1999; 12(12): 1035-40.

39 Grassi G, Maccaroni P, Meyer R, Kaiser H, D'Ambrosio E, Pascale E et al. Inhibitors of DNA methylation and histone deacetylation activate cytomegalovirus promoter-controlled reporter gene expression in human glioblastoma cell line U87. Carcinogenesis 2003; 24(10): 1625-35.
40 Krishnan M, Park JM, Cao F, Wang D, Paulmurugan $\mathrm{R}$, Tseng JR et al. Effects of epigenetic modulation on reporter gene expression: implications for stem cell imaging. FASEB J 2006; 20(1): 106-8.

41 Liu HS, Jan MS, Chou CK, Chen PH, Ke NJ. Is green fluorescent protein toxic to the living cells? Biochem Biophys Res Commun 1999; 260(3): 712-7.

42 Rols MP, Delteil C, Golzio M, Dumond P, Cros S, Teissie J. In vivo electrically mediated protein and gene transfer in murine melanoma. Nat Biotechnol 1998; 16(2): 168-71.

43 Wells JM, Li LH, Sen A, Jahreis GP, Hui SW. Electroporation-enhanced gene delivery in mammary tumors. Gene Ther 2000; 7(7): 541-7.

44 Bettan M, Ivanov MA, Mir LM, Boissiere F, Delaere P, Scherman D. Efficient DNA electrotransfer into tumors. Bioelectrochemistry 2000; 52(1): 83-90.

45 Cemazar M, Sersa G, Wilson J, Tozer GM, Hart $\mathrm{SL}$, Grosel A et al. Effective gene transfer to solid tumors using different nonviral gene delivery techniques: electroporation, liposomes, and integrin-targeted vector. Cancer Gene Ther 2002; 9(4): 399-406.

46 Cemazar M, Wilson I, Dachs GU, Tozer GM, Sersa G. Direct visualization of electroporation-assisted in vivo gene delivery to tumors using intravital microscopy - spatial and time dependent distribution. BMC Cancer 2004; 4(81.) 